\title{
Transformational and Transactional Leadership Styles on Organisational Change in the United Arab Emirates
}

\author{
Abdelkarim Fuad Kitana * \\ City University College of Ajman, United Arab Emirates
}

\begin{abstract}
Leaders play essential roles in developing an organisation. The underlying factors of the organisational mechanism, such as process-oriented systems, motivation, and vision, are provided by the leader. Therefore, the study aims to identify and examine the role of transactional and transformational leadership styles in the organisational change process. The researcher believes that those styles of leadership are essential in achieving a positive change in organisations. A focus group session of 120 participants, consisting of subordinates and employees from varying and diverse organisational backgrounds, has been conducted and the study found that certain leadership qualities and attributes are valued over others. Transactional and transformational leadership styles need to be integrated for the success of the organisation. Their association and relationship with factors such as organisational knowledge management, attitudinal and perceptual changes in employee perspectives, cultural changes, development in productivity and efficiency are also among the factors of organisational success. This paper looks at how the leadership styles are associated with each other, along with the reasons why each of these styles might be relevant in their way depending upon circumstances and organisational objectives concerning change and restructuring. The study showed that employees in the private sector prefer the transformational style rather than the transactional style.
\end{abstract}

Keywords: Leadership, Transformational Leadership, Transactional Leadership, Organizational Change.

JEL Classification: D21, L39

Paper Type: Research

\section{INTRODUCTION}

Leaders play one of the most central roles in the overall functioning and continual development of an organisation. The leader provides essential core elements of the organisational mechanism, such as process-oriented systems, motivation, and vision in an institution or establishment. Another essential role that the leader plays within an

\footnotetext{
*Corresponding author: E-mail: trainer_2012@hotmail.com
} 
organisation is the management of knowledge and the use thereof for competitive advantage. All of these aspects are used as fundamental reference points for understanding how transactional and transformational leadership styles influence organisational growth and motivation levels amongst employees.

The first mention and outlining of these two primary forms of leadership styles were made by Burns (1978) in his famous work titled "Leadership," which has been since hailed as an excellent read for everyone who ventures into the domain of studies concerning organisational management and leadership styles in general. According to him, leaders who followed the transactional style of managing organisational functions utilised and leveraged the immediate self-interests of employees as the core motivating factor. On the other hand, the leaders who were conditioned by the transformational style leveraged the ability to uplift employee morale and cater to the moral obligations of employees in order to make them work efficiently.

As the years have gone by, the need to increase the involvement of employees and pursue a more inclusive means of organisational management has emerged as a core aspect of organisational growth and change. Under this scenario, leaders had to shoulder the responsibility of developing teams that consisted of highly motivated individuals who could not merely be made to adhere to responsibilities through organisational commitment. This further involved the development of values centred on not only quantity of output but furthermore, quality of service as well as cost-effectiveness of production. The Cold War era was a significant time of upheaval into this form mentioned above of organisational functioning because, during this age, the flexibility of teams and management styles was hailed as the most crucial factor. The age ushered in the automation of less-skilled jobs and organisational responsibilities, coupled with the outsourcing of less-skilled functions to third-world countries. Those willing to work and add value to the organisation had to focus on developing specific abilities and skills through education and increased experience (Bass, 1999).

Today, with the rise of concepts such as organisational citizenship behaviour (OCB), which instills the urge to go beyond and above the norms of organisational functioning in order to add value to the enterprise, transformational leadership has emerged as the more deployed form of management of employee interests. However, there are times when transactional leadership styles have resorted in order to hasten the process of organisational growth. This paper will be looking at both secondary data as well as primary data in order to build a holistic understanding of how these two leadership styles influence organisational change. There are a few factors that will be used as reference points for this comparison and analysis of the impact of these leadership styles on change and growth within an enterprise. These factors will include the management of organisational knowledge, financial and attitudinal outcomes, and organisational innovation.

\section{LITERATURE REVIEW}

Before we delve into the intrinsic factors that can help us understand the subtle, as well as the apparent distinction in the medium and extent of influence of leadership styles on organisational change, specific definitions, and contextual elements, need to be made clear. The delineation of leadership styles, as per Burns (1978), opened up various perspectives and angles through which leaders and their impact on organisational functioning might be observed and analysed. This delineation was affected by the definition of a leader as a transformational force within the organisation rather than a position associated with rewards and control, which was the prevalent view in the earlier era of the industrial revolution. 
According to Burns (1978) and Bass (1985), a transformational leader established himself/herself as a role model for other employees by setting examples that they could look up to as standards or ideals for self-growth and responsibility management. This process of example-setting virtually encompassed the leader's intent of gaining the trust of the employees and winning their confidence. The focus in this scenario is the set future goals and build expectations that the employees have to live up to in order to achieve said goals. The qualities mentioned under the umbrella of such a leadership style have also been the focus of study for numerous other authors, such as Conger \& Kanungo (1998), who labelled this style as charismatic leadership.

Researchers such as Burns (1978) and Avolio (1999) looked at the other side of the coin that defined transactional leadership styles, a common form of leadership during the autocratic age of organisational functioning that the era of the industrial revolution was defined. The fundamental definition of such a leadership style was the premise of establishing exchange relationships with employees by leaders in order to appeal to their self-interests. The fundamental elements of such a leadership style involved the clarification of roles and responsibilities of employees, the use of rewards as incentives for meeting task-based goals and objectives, and the use of corrective methods when employees do not meet these goals.

The research into leadership styles, however, dates back even further than the seminal studies that helped in establishing the definitions of these two basic leadership styles. It was the author Bales (1950) who set the stage for the delineation of these two styles by defining two forms of opposing means of organisational management, namely task-oriented and interpersonal management of employee effectiveness and motivation. Further developed in terms of applicability and implications by authors like Likert (1961) and Hemphill \& Coons (1957), these management styles were defined by how leaders chose to associate with employees and align them towards the interests of the organisation.

On the one hand, the task-oriented style of employee management saw leaders who invested their time and energy into ensuring that task-oriented responsibilities on employees have adhered. This involved organising employee roles in the form of taskbased activities that were relevant to the organisation as a whole. On the other hand, the leadership style, which was defined as the interpersonal style of management involved the impetus on the leader to develop strong interpersonal relationships with employees to cater to their moral and emotional well-being.

This delineation in and of itself has been influenced by and molded into varying forms of defining styles, such as autocratic and democratic leadership styles, the definitions of which were borne through experimental studies conducted by Lewin and Lippitt (1938) and further developed by authors like Vroom and Yetton (1973), who defined these styles according to the degree of employee participation that was allowed by leaders in the organizational decision-making process. Fundamentally, the democratic leadership style saw leaders allowing for a significant degree of employee participation in the decisionmaking process, while the severe form of management almost always restricted the decision-making process to the leaders themselves, the employees having no or little say in the matter.

The historical and traditional development of the definitions of these styles shed enormous amounts of light into their inherent effectiveness in terms of organizational change as well as the core aspects which contribute to their relevance in the present age, thereby defining the nature and extent of their effectiveness within the arena that we have chosen to enter through this research paper. In order to delve further into the subject of effectiveness and extent of influence of these two styles on organisational change, we 
shall be considering specific fundamental reference points within the organisational machinery by which we can estimate the same.

\subsection{Organisational Knowledge}

One of the most procedurally efficient and effective ways to observe the impact of each of the leadership styles on organisational change is to use the concept of organisational knowledge management as a reference. The strategic management of organisational knowledge is one of the most basic means of organisational change, growth, and development. When endeavouring to look at how organisational knowledge is managed under differing leadership styles, the first step is to define what this form of knowledge is.

According to Boisot (1998) and Grant (1996), organisational knowledge is encompassed in all the tacit as well as explicit forms of knowledge which are possessed by the individuals within an organisation. This includes the knowledge that is written down or codified in information systems, databases, and manuals on the one hand, and processes, systems, and products on the other. Moreover, the capsule organisational knowledge also contains within it the social processes, routines, and cultural norms that define the collective level of integrity and structure within the organisation.

Authors like Spender (1996) and Argote, McEvily, and Reagans (2003) further differentiate between the types of knowledge that are encompassed within an organisational system, such as personal and social knowledge, as well as public and private knowledge. Once we have defined knowledge in an organisational context, we further understand that said knowledge can be created, shared, and exploited. When it comes to creating knowledge within the organisation, leaders play a huge role in providing the right creative and functional direction for workers involved in the creation process.

The leader sets the context for knowledge creation amongst the employees further plays a massive role in defining the overall levels of creativity that is expressed in the organisation (Mumford, Whetzel, \& Reiter-Palinon, 1997). Under these circumstances, leader ultimately impacts the very nature of creative behaviour under the organisational roof by defining the types of behaviour that are rewarded, the behavioural patterns that are shunned as well as the rewards that are offered for following or embodying the "right" behavioural traits.

When it comes to sharing knowledge, the leader is responsible for creating a climate or environmental context that allows for increased receptiveness towards new ideas. Facilitating the process of knowledge sharing through periodic meetings and transactions between employees is a core factor that comes under the leader's repertoire of responsibilities. Moreover, setting the tone and the climate for these sharing sessions is also a fundamental responsibility of the leader, which cannot be ignored. For instance, employees may be more willing to share knowledge when they are praised by their seniors and may also be coerced into coming up with new ideas when there are rewards associated with the process (Bryant, 2003).

According to Bryant (2003) study, a reliance on one leadership style in order to ensure that something as fundamental to organisational processing and growth as inherent knowledge is managed skillfully and to the best of enterprise ability would be a severe miscalculation. This is because a blend of transactional and transformational leadership styles ensures a balance between creativity and process-oriented results, or in other words, chaos and order.

There have been several times when transformational leadership has considerably improved the overall degree of novel and new ideas within the organization, but due to the lack of transactional leadership to continue and progressively build upon the process of 
coming up with new ideas and converting them into products, organizations have failed to capitalize on the power of this priceless resource of knowledge.

\subsection{Organisational Innovation}

A natural by-product of the progression from knowledge creation to exploitation stably and positively is innovation. Hence, looking at the correlation between innovation and leadership styles within an organisation could be a powerful indicator of how knowledge as an asset is dependent upon creative climate and employee perceptions, which are primarily facilitated by the leader. According to Jung, Chow, \& Wu (2003), the role of transformational leadership in empowering employees to innovate beyond the actual norms and boundaries of accepted responsibility is one of the most significant correlations that exist within the organisational framework. As mentioned above, a balance between the two leadership styles facilitates proper management of organisational knowledge. However, when it comes to explicitly defining the relationship leadership styles and innovation, transformational leadership styles are much more effective as compared to transactional styles of management. The authors built the bridge between transformational leadership style and innovation through a consideration of the impact that this particular style had on organisational climate as well as employee empowerment. Based on the findings from 32 Taiwanese companies, the authors were able to observe that transformational leadership styles allowed for the development of a more cohesive and holistic organisational climate as far as innovation and support for ideas on innovation were concerned.

This is in direct correlation to the studies conducted by authors like Sosik (1997), who mentioned that specific types of leadership styles, such as those which consistently challenge employees to be creative and to think outside the box, allowed for a palpably more conducive environment or organisational climate for innovation.

At the same time, the authors also found a flipside to the use of transformational leadership styles within an organisation. In this case, other than the impact that this type of management style had on innovation and organisational climate, another bridge was built between the two factors by looking at how transformational leadership styles influenced employee empowerment. In this case, even though there was a definite positive correlation between employee empowerment and the use of transformational styles of management by leaders, it was found that there was a negative influence that the increased levels of empowerment had on the levels of innovation within the organisation. In other words, the level of innovation is low in organisations where autonomy was attributed to employees as compared to the transactional leadership styles, where the innovations were derived from the form of process-oriented practice.

\subsection{Attitudinal and Financial Outcomes}

According to Weber, Kelloway, and Barling (1996), the use of transformational leadership styles within an organisational context has an immense impact on the perception harboured by subordinates as to the atmosphere of control and responsibility that they are placed within. In other words, the subordinates who were subject to the study proceeded to rate their leaders higher on the scale of individual consideration, charisma, and intellectual stimulation when a transformational form of leadership style was employed. This shows a definite shift in the attitudinal perceptions and perceptions that employees harbour towards their leaders based on the style of management chosen by the top management. 
Further results of the study also showed that the higher the leaders were rated on the charisma, individual consideration, and intellectual stimulation scales, the higher was the degree of organisational commitment that was shown by the employees/subordinates themselves. Moreover, the results also showed support for the influence of this style of leadership on the overall level of economic output on organisational branches or departments, which showed considerably more positive results when governed by this form of management.

These results serve to enhance the positive correlation that was found between transformational leadership styles and organisational effectiveness from the point-of-view of employee motivation, commitment, and contribution. These positive correlations were first outlined by Howell and Frost (1989) and Kirkpatrick and Locke (1996), who solidified the role of transformational relationships in changing the perceptions that subordinates had towards leaders and their associations with them towards a more positive sentiment.

As far as the financial outcome and its correlation with transformational leadership is concerned, Howell and Avolio (1993) found that the shifts from transactional to transformational styles exert a positive influence on financial performance.

\section{RESEARCH METHODOLOGY}

In order to gather the data for this study, a few series of focus group interviews have been conducted from different organisations such as banks, hospitals, and universities. The interview focuses on the employees from the first and middle management levels. The purpose of the interview is to understand how they are comfortable in the working environment that consisted of both leadership styles.

The questionnaire consisted of 8 questions, each of them signifying specific circumstances that employees might find them in during their daily routine. The session aimed to facilitate discussion amongst employees from different work cultures and climates, thereby ensuring that the results that come out of the session are not organisation-specific, thereby allowing for a glimpse into the influence of leadership styles beyond the purview of enterprise-oriented boundaries.

Notes were taken throughout the session to ensure that all essential points and arguments were taken into consideration. Special care was taken in order to ensure that the focus group members chosen for the session were subordinates that consisted of some form of hierarchy and had inculcated the new age aspects of innovative work culture and community-based employee motivation and inclusion. The number of participants chosen for the session was 120 from various organisational backgrounds.

In order to deal with this, the participants were allowed to get acquainted with each other before the session, while the interaction was also promoted after the session as a way of getting them acclimatised to each other and to the environment that was constructed to facilitate the session itself. This method helped in allowing the members to have their say and to be willing enough to speak out their own experiences during the session, an aspect which was further promoted by due intervention during questions in order to ensure that everyone had a say in each circumstance/question. The questions themselves were structured in such a way that lengthy and articulate discussions could be avoided, and the participants could more or less summarise their views and opinions straightforwardly.

All surveys were collected at different levels of management posts. Participants were scheduled to attend a data collection session during a 1- to 3-days period based on their schedules. After explaining the purpose of the study and the protections for anonymity, we gave all participants the option of sitting quietly and not participating in the study. All survey scale items used in the current study were first examined and suitably modified in 
discussion with experts and consultants. However, relatively few changes were required to the original survey instruments, including the MLQ; in which only two items were changed on the Management by Exception Scale (that is, "Concentrates full attention on dealing with mistakes, complaints, and failures" and "Directs attention toward failures to meet standards"). There were 120 leadership items measured in each MLQ - Form survey. Participants rated one of their respective leaders.

\section{DATA ANALYSIS}

The results are divided into each of the questions that were presented to the group participants and how these circumstances were considered and used as a reference for outlining their perspectives towards management styles. The first few questions, as one will notice, as to reach towards the core subject of leadership and management styles within the organisation, while the ones that follow are directed towards specific aspects that pertain to the impact of these styles, such as organisational knowledge management, and innovation.

1. When doing everyday tasks, are you more likely to think outside the box or focus on basic deliverables by using routine practice?

The answer to this question was seen as a way to understand the basic leadership styles that might be more prevalent amongst the employees in their own organisational culture. A majority of the participants, about $40 \%$ in total, mentioned that they follow routine procedures most of the time for daily tasks, even though innovation was an aspect that they were involved. The reasoning that was given for this particular answer varied from the fact that deadline-based tasks required routine practice in order to achieve completion as rapidly and efficiently as possible to the fact that they were not introduced to any new and unique ways of completing their daily tasks. The $60 \%$ individuals who said that they had the resources and the time to think outside the box and bring in novelty even during daily tasks mentioned that they were encouraged and even challenged to do so in their organizations, as this was one of the main ways in which their enterprise functioned, thereby portraying institutions that had a powerful impetus on knowledge creation amongst employees.

2. In your organisation, are new ideas rewarded, or do they often go through layers of scrutiny and modification in order to fit the organisational mould of procedure and standards?

This question incited answers, which showed that most of the organisations that the employees were coming from consisted of a transactional form of leadership. About $63 \%$ of participants mentioned that new ideas in their organisation were not rewarded and were censored and moulded in order to fit into the already existing constructs. The other mentioned that there was some reward system in place that encouraged them to come up with new ideas, even though the resources and the motivation behind coming up with novel concepts were not always pronounced or established.

3. How is a failure at your duties reprimanded within the organisation?

This question saw many answers, including pay cuts, suspension, and demotion. However, there were milder answers as well, including counselling sessions and reconciliation interviews, which were directed at finding the root cause of the problem that 
led to the failure. This showed that there was a definite mix of organisational climates amongst the participants, which covered the entire spectrum of leadership styles.

4. How willing and confident would you be in suggesting a change in the method as far as your role and responsibility in the organisation is concerned?

This question was aimed at understanding how easy or difficult it was for employees to change their medium or method of working within the organisation, thereby allowing a glimpse into the readiness with which their respective organisations dealt with change and restructuring. As expected, more than $55 \%$ of the participants mentioned that their opinions might not be taken into consideration or that they were happy with the method of work that they were following at the moment. The minority, which consisted of about 5 participants, mentioned that their work culture either encouraged or allowed for healthy discussions on procedural changes and alterations periodically.

5. If you had the chance to lead a team of your own within your organisational framework, what sort of communication method would you utilise as far as team members sharing their ideas and daily work reports are concerned?

In this case, it was observed that the individuals who came from a transactional background preferred message boards and digital means of communication, while those who came from more liberal, transformational backgrounds preferred more informal styles of communication, such as presentations, one-to-one sessions, and group sessions. This shows that how the organisational culture facilitates communication of ideas is different under varying leadership styles, with one emphasising rapidity and efficiency while the other placing impetus on intimacy and communal harmony.

6. If you were to grade senior officials based on their competency, what would be the main attributes that you would select for the same?

In this case, the answer served to offer a glimpse into both the qualities that subordinates look for in their leaders as well as some of the attributes that they incorporate into an ideal persona associated with a leadership position. Some of the main attributes that were mentioned included a strong personality, calm mannerisms, originality, or uniqueness of perspective, friendly and approachable, and open to new ideas. The most exciting aspect associated with the answer to this question was that many of the attributes that were mentioned overlapped amongst employees from both transactional and transformational leadership backgrounds. This meant that there was common perceptual ground amongst employees in all organisations which denoted a good leader, many of whose traits were related to the transformational style of leadership.

7. If you had to build a routine practice in your organisation that employees could follow, what would this practice be oriented towards?

This question was aimed at placing the participants, all of whom were subordinates or employees, in the shoes of a leader. The answers to this question showed how the organisational climate and the prevalent leadership style in their enterprise impacted their perceptions of their leadership traits and preferences. For instance, the individuals who came from transformational leadership climates mentioned that they would create procedures and processes that were directed towards employee empowerment, innovation, progressive thinking, and cultural developments within the organisation. On 
the other hand, the employees who came from transactional leadership climates attributed aspects like enhancement of existing procedures, improving efficiency and enhancing production capacity as their primary objectives behind integrating new procedures in the organisation.

8. If you had the chance to change one thing in your leader's or senior's personality, what would it be?

As a response to this question, the participants showcased answers that showed some of the primary leadership attributes that they perceived as being detrimental to their levels of organisational loyalty and commitment. In this case, the attributes that were mentioned included short-temper, lack of involvement, decreased interest in employee participation, and even lack of confidence in decision-making. All of these answers again overlapped between employees from transactional and transformational leadership backgrounds, showing a common-ground as far as leader perceptions were concerned.

\section{CONCLUSION AND RECOMMENDATIONS}

Some of the main findings and conclusions that can be derived from the results include the crucial element that albeit the kind of organisational climate or culture that an employee comes from, specific leadership attributes contribute towards their ideal preference in both positive and negative domains. This means that there is a standard perceptual reference that individuals derive their understanding of a good leader. In the case of employees that come from highly transactional leadership style-backed organisational environments, the attributes often showcase a need to integrate more openness and inclusion of the leader and the employee in the decision-making process and the innovation process as a whole. As far as the employees from increasingly transformational management style-backed organisations are concerned, their preferred attributes for a leader stem from their experience with specific methods that seem to be working well for them already. In both these contexts, there are definite overlaps, meaning that a more transformational style of leadership might work well for all organisations owing to the age that we are in.

At the same time, the more crucial aspect of the findings pertains to the very definitions of transformational leadership and transactional leadership within the context of the age that we are in at the moment. The results show that even though there might be standard perceptual agreements amongst employees from different organisational backgrounds regarding leadership qualities, the type of mechanisms, procedures, or processes that these employees reinforce as positive and enhancing towards the organisation seem to be vastly different. Transactional leadership styles, even though being found wanting in terms of employee motivation to embrace novelty and innovation in general, have been successful in instilling them with respect for efficiency, capacity, quality, and production capabilities.

These aspects are as important in today's world, where innovation and technology are advancing at rates like never before, as it is in the world that people were used to during the industrial revolution. Hence, even though transformational leadership styles might be taking precedence over transactional leadership styles as far as employee involvement, workplace cultural development, ethical and moral maturity, and other aspects, transactional leadership styles hold merit for procedural and process-oriented organisational aspects that serve to improve efficiency and productivity.

The final findings or conclusion of the paper points to the fact that for organisational change, a balance between both transactional and transformational leadership styles is necessary. When change or restructuring is focused on cultural changes or modifications 
in terms of employee ethics and moral standards, transformational leadership styles are much more contextually relevant because interpersonal factors play a significant role in such changes. However, when organisational changes are focused on efficiency and productivity, transactional leadership styles can be much more effective as compared to the former. As studies have taught us, it is crucial to necessitate the balance between cultural and procedural changes for successful organisational restructuring, thereby facilitating the need to employ both transactional and transformational leadership styles depending upon the circumstances taken into consideration.

As per this understanding that has been developed through the findings, the essential recommendation is to build organisational structures that incorporate both the flexibility of transformational leadership as well as the efficiency-oriented design of transactional leadership styles. In order to truly master organisational change, leaders have to take control and manage the organisational knowledge as well as attitudinal, perceptual, and cultural elements within the organisation.

Moreover, both ethical and moral values need to be taken into consideration, on the one hand, to ensure that employees have the confidence as well as the drive to see the change and restructuring through. At the same time, using transactional leadership styles, leaders ought to invest their efforts and time into building processes and procedures which take efficiency and productivity into concern over and above employee empowerment to ensure that amid cultural values, the organisational requirements are not compromised.

Further research on the present topic of work-related to transformational and transactional leadership styles in achieving organisational change, more respondents from the local groups should be included. In many cases, the number of foreign involvement in the study proved to be too small to reach any significant conclusions. Furthermore, the most famous case was that the researcher strongly recommends future research to survey in one area or a similar group of works.

There are several limitations that we faced during and completing this research work; these were time constraints, geographical constraints, and financial constraints. This research was aimed to be completed in the limited time frame and consists of different total quality management tools which are being implemented in different organisational.

\section{REFERENCES}

Argote, L., McEvily, B., \& Reagans, R. (2003). Introduction to the special issue on managing knowledge in organizations: Creating, retaining, and transferring knowledge. Management Science, 49(4), v-viii.

Avolio, B. J. (1999). Full leadership development: Building the vital forces in organizations. Thousands Oaks, CA: Sage.

Bass, B. M. (1999). Two Decades of Research and Development in Transformational Leadership. European Journal Of Work And Organizational Psychology, 8 (1), 9-32.

Boisot, M. H. (1998). Knowledge assets: Securing competitive advantage in the information economy. New York: Oxford University Press.

Bryant, S. E. (2003). The Role of Transformational and Transactional Leadership in Creating, Sharing and Exploiting Organizational Knowledge. Journal of Leadership and Organizational Studies 9, no. 4, 32-44.

Burns, J. M. (1978). Leadership. New York: Harper \& Row.

Conger, J. A., \& Kanungo, R. N. (1998). Charismatic leadership in organizations. Thousand Oaks, CA: Sage.

De Jong, J. P., \& Den Hartog, D. N. (2007). How leaders influence employees' innovative behaviour. European Journal of innovation management, 10(1), 41-64.

Grant, R. (1996). Toward a knowledge-based theory of the firm. Strategic Management Journal. 17 (Winter Special Issue), 109-122. 
Howell, J. M., \& Avolio, B. J. (1993). Transformational leadership, transactional leadership, locus of control, and support for innovation: Key predictors of consolidated-business-unit performance. Journal of Applied Psychology, 78, 891-902.

Howell, J. M., \& Frost, P. J. (1989). A laboratory study of charismatic leadership. Organizational Behavior and Human Decision Processes, 43, 243-269.

Judge, T. A., \& Piccolo, R. F. (2004). Transformational and transactional leadership: a metaanalytic test of their relative validity. Journal of applied psychology, 89(5), 755.

Jung, D. I., Chow, C., \& Wu, A. (2003). The role of transformational leadership in enhancing organizational innovation: Hypotheses and some preliminary findings. The Leadership Quarterly 14, $525-544$.

Kirkpatrick, S. A., \& Locke, E. A. (1996). Direct and indirect effects of three core charismatic leadership components on performance and attitudes. Journal of Applied Psychology, 81, 3651.

Mumford, M. D., Whetzel, D. L., \& Reiter-Palinon, R. (1997). Thinking creatively at work: Organization influences on creative problem-solving. Journal of Creative Behavior. 31, 7-17.

O'Reilly, C. A., Caldwell, D. F., Chatman, J. A., Lapiz, M., \& Self, W. (2010). How leadership matters: The effects of leaders' alignment on strategy implementation. Leadership Quarterly, 21(1), 104-113.

Sosik, J. J. (1997). The Dream Weavers: Strategy-focused leadership in technology-driven organizations. Greenwich, CT: Information Age.

Spender, J. C. (1996). Making knowledge the basis of a dynamic theory of the firm. Strategic management journal, 17(S2), 45-62.

Weber, T., Kelloway, E. K., \& Barling, J. (1996). Effects of Transformational Leadership Training on Attitudinal and Financial Outcomes: A Field Experiment. Journal of Applied Psychology, 81(6), 827-832.

West, M. A., Borrill, C. S., Dawson, J. F., Brodbeck, F., Shapiro, D. A., \& Haward, B. (2003). Leadership clarity and team innovation in health care. The leadership quarterly, 14(4-5), 393410.

Zhang, X., \& Bartol, K. M. (2010). Linking empowering leadership and employee creativity: The influence of psychological empowerment, intrinsic motivation, and creative process engagement. Academy of management journal, 53(1), 107-128. 